\title{
CONFORMALLY FLAT 3-MANIFOLDS AND EUCLIDEAN POLYHEDRA.
}

\author{
SER PEOW TAN
}

\begin{abstract}
In this paper, we study the deformation space $\mathcal{C}(M)$ of flat conformal structures on a compact hyperbolic 3-manifold $M$ by using a geodesic simplicial decomposition $\Delta$ of $M$. In particular, we demonstrate the relations between flat conformal structures, singular pleated hyperbolic structures composed of ideal tetrahedra and euclidean polyhedra.
\end{abstract}

\section{Introduction AND Preliminaries}

A flat conformal structure (f.c.s.) on a $n$-dimensional manifold $M$ is a maximal system of coordinate charts based on $S^{n}$ such that locally, the coordinate changes are local conformal diffeomorphisms of $S^{n}$. For $n \geq 3$, by Liouville's theorem, any local conformal diffeomorphism of $S^{n}$ is induced by a global Möbius transformation so a f.c.s. on $M$ is also called a Möbius structure. This is a $(G, X)$ structure in the sense of Thurston [22] with the model space $X=S^{n}$ and the transformation group $G=\operatorname{Möb}\left(S^{n}\right)$. A f.c.s. is also equivalent to the conformal class of a conformally locally Euclidean metric on $M$. Such structures are important in many different contexts, for example, as a class of structures, they naturally contain the spherical, similarity and hyperbolic structures. The literature on such structures is extensive, see for example [2], [7], [13], [14], [17] and the references contained therein.

If $f: M \mapsto N$ is a covering map and $N$ has a f.c.s. then we can pull-back the f.c.s. on $N$ to obtain a f.c.s. on $M$. If $M$ has a f.c.s. to start with, we say that the map $f$ is a conformal map if the two structures on $M$ are the same. There are two natural equivalence relations on the set of all flat conformal structures on $M$ as follows: Given two f.c.s. on $M,\left(M, \rho_{1}\right)$ and 
$\left(M, \rho_{2}\right),\left(M, \rho_{1}\right) \sim_{1}\left(M, \rho_{2}\right)$ if there is a diffeomorphism from $M$ to itself which is a conformal map with respect to $\left(M, \rho_{1}\right)$ and $\left(M, \rho_{2}\right)$, and $\left(M, \rho_{1}\right) \sim_{2}\left(M, \rho_{2}\right)$ if there is a diffeomorphism from $M$ to itself isotopic to the identity which is a conformal map with respect to $\left(M, \rho_{1}\right)$ and $\left(M, \rho_{2}\right)$. The space of f.c.s. on $M$ modulo $\sim_{1}$ is called the moduli space of f.c.s. on $M$ while the space of f.c.s. on $M$ modulo $\sim_{2}$ is called the deformation space or Teichmuller space of f.c.s. on $M$ which we denote by $\mathcal{C}(M)$. Clearly, the moduli space is obtained from the Teichmuller space by the action of $\operatorname{Diff}(M) / D$ if $f_{0}(M)$. The elements in $\mathcal{C}(M)$ are called marked f.c.s. on $M$.

The aim of this paper is to study the space $\mathcal{C}(M)$ in the case when $M$ is a closed oriented 3-manifold admitting a hyperbolic structure. Note that in this case, the space $\mathcal{C}(M)$ contains $\mathcal{T}(M)$, the space of hyperbolic structures on $M$. By the celebrated Mostow rigidity theorem [18], $\mathcal{T}(M)$ is trivial, however, the space $\mathcal{C}(M)$ need not necessarily be trivial. The first non-trivial deformations of f.c.s. on a closed hyperbolic $n$-manifold $(n \geq 3)$ was constructed by Apanasov in [1]; subsequently, Kourouniotis [12] and Johnson and Millson [7] generalised a bending construction of Thurston's to construct non-trivial deformations of f.c.s. on manifolds $M$ admitting totally geodesic hypersurfaces. In particular, Johnson and Millson were able to construct examples of hyperbolic $n$-manifolds $(n \geq 4)$ whose deformation spaces $\mathcal{C}(M)$ are singular. They did this by constructing manifolds $M$ which have intersecting totally geodesic hypersurfaces (bending can be carried out independently along nonintersecting totally geodesic hypersurfaces) and using group cohomology to show that there are obstructions to bending simultaneously along both intersecting hypersurfaces. This provides a striking contrast to the dimension 2 case where $\mathcal{C}(M)$ is the deformation space of $\mathbf{C P}^{1}$-structures on a closed oriented hyperbolic surface $M$ of genus $g \geq 2$ and $\mathcal{C}(M)$ is homeomorphic to $\mathbb{R}^{12 g-12}$. The case when $n=3$ is particularly interesting, the obstructions of Johnson-Millson vanish and it is not clear if it is possible for $\mathcal{C}(M)$ to be singular when $M$ is a closed oriented hyperbolic 3-manifold.

The first non-bending deformations were constructed by Apanasov (see [2]) using a pea-pod group construction, he called these 'stamping' deformations. Using the maximal ball technique, we independently constructed and gener- 
alised these deformations in [20]; in fact we showed that both bending and stamping deformations were generalisations of Thurston's bending construction for dimension 2 and that in higher dimensions, it is sometimes possible to bend along intersecting totally geodesic hypersurfaces as long as a spherical polygonal condition was satisfied by the bending parameters and dihedral angles in a link of the intersection. Furthermore, the underlying structure can now be deformed to cone hyperbolic structures with cone singularities at the intersection of the hypersurfaces. However, none of these constructions gave the complete local picture of $\mathcal{C}(M)$. The only complete results we know of in this case are results of $M$. Kapovich [10] where he showed that $\mathcal{C}(M)$ is locally trivial about the hyperbolic structure for infinitely many hyperbolic manifolds $M$ obtained by hyperbolic Dehn surgery on hyperbolic two bridge knots.

In this paper, we present a different approach to this problem using the following facts:

(1) There exists a geodesic simplicial decomposition of $M$;

(2) For any four distinct points in $S^{3}$ in general position, there is a unique sphere passing through all four points so that the convex hull of the four points in $\mathbf{H}^{4}$ is an ideal tetrahedron which lies on a totally geodesic hypersurface in $\mathbf{H}^{4}$;

(3) Combining (1) and (2), we can associate to a quasi-Fuchsian structure on $M$ a singular pleated hyperbolic structure made up of ideal tetrahedra pieces;

(4) The singular pleated hyperbolic structure of (3) determines and is determined by the induced singular pleated euclidean structures on the links of the vertices, up to similarity, in other words, euclidean polyhedra.

To state our result more precisely, we start with some definitions. Let $(M, \rho)$ be a closed oriented hyperbolic 3-manifold. It is well known that $(M, \rho)$ admits a geodesic triangulation, we fix one such triangulation and denote it by $\Delta$. Suppose further that there are $k$ vertices and $m$ tetrahedra in the triangulation $\Delta$. Let $\mathcal{C}(M, k)$ denote the deformation space of marked f.c.s. on $M$ with $k$ distinguished points. The hyperbolic structure on $M$ together with $\Delta$ determines a point $\left(\rho ; v_{1}, \ldots, v_{k}\right) \in \mathcal{C}(M, k)$ where $v_{1}, \ldots, v_{k}$ are the vertices 
of $\Delta$. Note that there is a natural projection map $p: \mathcal{C}(M, k) \mapsto \mathcal{C}(M)$ which 'forgets' the distinguished points; the fibre of the map has real dimension $3 k$.

If $v_{i}$ is a vertex of $\Delta$, let $L\left(v_{i}\right)$ be the link of $v_{i}$, it is topologically a sphere and the triangulation $\Delta$ induces a triangulation $\Delta\left(v_{i}\right)$ of $L\left(v_{i}\right)$. We have the following:

Lemma 1. Let $(M, \rho)$ be a closed orientable hyperbolic 3-manifold and let $\Delta$ be a topological triangulation of $M$ isotopic to a geodesic triangulation with $k$ (fixed) vertices $v_{1}, . ., v_{k}$. Then the hyperbolic structure $\rho$ on $M$ together with the triangulation $\Delta$ determines the following:

(a) a distinguished point $\left(\rho ; v_{1}, \ldots, v_{k}\right) \in \mathcal{C}(M, k)$;

(b) a singular pleated hyperbolic structure $M_{\rho, \Delta}$ on $M$ composed of ideal tetrahedra with pleating locus contained in the faces of $\Delta$; and

(c) a set of $k$ euclidean polyhedra $P_{i}$ up to similarity which are the structures induced by $M_{\rho, \Delta}$ on the links $L\left(v_{i}\right)$.

More generally, we have the following:

Theorem 1. The triangulation $\Delta$ determines a local homeomorphism beween the following three spaces:

(a) the space $\mathcal{C}(M, k)$ about $\left(\rho ; v_{1}, \ldots, v_{k}\right)$;

(b) the deformation space of singular pleated hyperbolic structures on $M$ composed of ideal tetrahedra with pleating locus contained in the faces of $\Delta$, about the point $M_{\rho, \Delta}$; and

(c) the deformation space of $k$ euclidean polyhedra up to similarity about the point $\left(P_{1}, \ldots, P_{k}\right)$ satisfying the following conditions:

(i) each polyhedron is combinatorially equivalent to $\Delta\left(v_{i}\right)$.

(ii) the bending angle at an edge of $P_{i}$ is equal to the pleating measure on the corresponding face of $\Delta$; and

(iii) if $F_{1}$ and $F_{2}$ are two faces of the (not necessarily distinct) polyhedra $P_{i}$ and $P_{j}$ and $F_{1}$ and $F_{2}$ are in the links of vertices of the same ideal tetrahedron piece $T$ in $\Delta$, then $F_{1}$ and $F_{2}$ are similar triangles with appropriate identification of their vertices induced by labelling opposite edges of $T$ with identical labels . 
Much of our construction generalises to higher dimensions. One starts with a geodesic simplicial decomposition of the manifold $M$ and use the fact that $n+1$ points in general position in $S^{n}$ determine a unique $S^{n-1}$ sphere passing through all the points so that the convex hull of the $n+1$ points in $\mathbf{H}^{n+1}$ is a totally geodesic ideal simplex of dimension $n$. However, it seems that in the dimension 3 case, there is much greater hope of doing the explicit computations since the problem is now reduced to studying euclidean polyhedra. Unfortunately, a rough dimension count shows that there are in general, the same number of relations and parameters in the space of such polyhedra satisfying the conditions of (c) in theorem 1 so that to obtain concrete information about $\mathcal{C}(M)$, it seems necessary to study the equations arising from some triangulation in greater detail. In dimension two, the above approach gives local coordinates for $\mathcal{Q}_{g, 1}$, the space of quasi-fuchsian structures on a closed, orientable surface of genus $g \geq 2$ with one distinguished point, see [21].

The rest of this paper is organised as follows: In $\S 2$, we define singular pleated hyperbolic structures and show how such structures can be obtained from a triangulation of $M$ with a flat conformal structure close to the hyperbolic structure, giving part (b) of lemma 1 (part (a) is obvious) and the correspondence between (a) and (b) of theorem 1 . In $\S 3$, we study in greater detail ideal tetrahedra which are the basic building blocks of the singular pleated hyperbolic structures and complete the proof by showing the correspondence between (b) and (c) of lemma 1 and theorem 1 .

Acknowledgement. The author would like to thank J. Millson, W. Goldman and M. Kapovich for their help with various queries, M. Van Loo for help with the diagrams and the Mathematics Institute at the University of Warwick for its generosity and hospitality during a stay in Spring, 1993 when part of this work was carried out.

\section{Singular PLEATED HYPERBoliC STRUCTURES}

Let $S$ be a complete orientable cone hyperbolic 3-manifold and $X \subset S$ be the discrete set of singular points of co-dimension 3 in $S$ (see [23] for the rigorous definition of a cone manifold and the codimension of the singular points). For example, if $S$ is obtained by a (compact) hyperbolic polyhedron by isometric 
pairing of faces, then the points on the edges would be singular and have codimension 2 if the total cone angle about the edge is not equal to $2 \pi$ and the vertices which lie on the intersection of singular edges would be singular and have codimension 3 . If the hyperbolic polyhedron is non-compact and has ideal vertices so that some of the edges are infinite, then we must ensure that the holonomy about an infinite edge has no translation factor. If the link of an ideal vertex is a sphere, then it is still considered to be a singular point of codimension 3, we call such singular points ideal.

A singular pleated hypersurface in a oriented hyperbolic 4-manifold $N$ is a complete cone hyperbolic 3-manifold $S$ together with a continuous map $\psi:\{S-X\} \mapsto N$, where $X$ is the set of singular points in $S$ of codimension 3 , which satisfies:

(a) $\psi$ is isometric, that is, every geodesic segment in $S-X$ is taken to a rectifiable arc in $N$ which has the same length;

(b) for each point $x \in S-X$, there is at least one open geodesic segment $\alpha_{x}$ through $x$ which is mapped to a geodesic segment in $N$.

The cone-hyperbolic structure on $S$ together with the map $\psi$ is called a singular pleated hyperbolic structure on $S$. By passing to the universal cover, we see that it is independent of $N$. The notion of the developing map and holonomy representation extends to singular pleated hyperbolic structures, the developing map is now a pleated immersion from $\tilde{S}-\tilde{X}$ to $\mathbf{H}^{4}$ and the holonomy representation maps $\pi_{1}(S)$ into $\operatorname{Isom}\left(\mathbf{H}^{4}\right)$.

If $x \in S-X$ and $m$ is the dimension of the span of the geodesic segments $\alpha_{x}$ satisfying (b), then the co-dimension of $x$ is defined to be $3-m$.

Note that a component of the set of points with co-dimension zero is an isometrically imbedded totally geodesic hypersurface in $N$, possibly with boundary. The pleating locus, denoted by $\mathcal{P} \mathcal{L}$ is the set of points with co-dimension 1. If $V$ is a component of $\mathcal{P L}, V$ is two-sided, denote the two sides by $U$ and $W$. The pleating measure on $V$ is defined to be the angle between the normal vectors to $U$ and $W$ (with direction determined by the orientations of $U$ and $W$ ) in $N$ at any point $x \in V$. More generally, one can define a transverse measure on the pleating locus (see [4], [9] or [14] for example) but for the purposes of this paper, the transverse measure will always be discrete 
and the above definition suffices. The pleating locus $\mathcal{P} \mathcal{L}$ together with the codimension 2 and 3 singular points can be thought of as a singular geodesic lamination on $S$, the transverse measure makes it a singular measured geodesic lamination, thus a singular pleated hyperbolic structure on $S$ can be thought of as a cone hyperbolic structure on $S$ together with a compatible singular measured geodesic lamination on $S$. As in the case of f.c.s. on $S$, a pleated hyperbolic structure on $S$ is defined up to diffeomorphisms of $S$ to itself.

In general, the topology on the space of singular pleated structures on $S$ is that induced by the developing map, i.e., two singular pleated structures are close to each other if the corresponding developing images of a fundamental domain are close in $\mathbf{H}^{4}$, up to conjugation by an element of $\operatorname{Isom}\left(\mathbf{H}^{4}\right)$. In the case where we are only considering the subspace of singular pleated structures where the pleating locus is contained in the faces of a fixed triangulation $\Delta$ of $S$, the topology is the same as that induced by the pleating parameters and the parameters for the tetrahedra pieces of $\Delta$.

Associated to a closed orientable hyperbolic 3-manifold $M$ and a geodesic triangulation $\Delta$ of $M$ into hyperbolic simplices is a singular pleated hyperbolic structure on $M$ where all codimension 3 singular points are ideal. This is constructed as follows:

Let $\left\{v_{1}, \ldots, v_{k}\right\},\left\{e_{1}, \ldots, e_{m+k}\right\},\left\{f_{1}, \ldots, f_{2 m}\right\},\left\{t_{1}, \ldots, t_{m}\right\}$ be the 0 -simplices (vertices), 1-simplices (edges), 2-simplices (faces) and 3-simplices (tetrahedra) of $\Delta$ respectively. If we perturb the positions of $\left\{v_{1}, \ldots, v_{k}\right\}$ slightly to $\left\{v_{1}^{\prime}, \ldots, v_{k}^{\prime}\right\}$, we get a geodesic triangulation $\Delta^{\prime}$ of $M$ which is combinatorially the same as $\Delta$, isotopic to $\Delta$ but geometrically different. Let $\tilde{M}$ be the universal cover of $M$ and let $\tilde{\Delta}$ be the triangulation of $\tilde{M}$ which is the lifting of $\Delta$.

Let $S^{3}$ be the unit sphere in $\mathbb{R}^{4}$, and $B^{4}$ the unit ball in $\mathbb{R}^{4}$ bounded by $S^{3}$. $B^{4}$ with the Poincare metric serves as a model for $\mathbf{H}^{4}$. The group of isometries of $\mathbf{H}^{4}$, Isom $\left(\mathbf{H}^{4}\right)$ can be identified with $\operatorname{Mob}\left(S^{3}\right)$. There are natural inclusions $\mathbf{H}^{3} \subset \mathbf{H}^{4}$ and $\operatorname{Isom}\left(\mathbf{H}^{3}\right) \subset \operatorname{Isom}\left(\mathbf{H}^{4}\right)$ where $\mathbf{H}^{3}=B^{4} \cap\left\{\left(x_{1}, x_{2}, x_{3}, x_{4}\right) \in\right.$ $\left.\mathbb{R}^{4} \mid x_{4}=0\right\}$.

Let $d e v$ and $\rho$ be the developing map and holonomy representation associated to the hyperbolic structure on $M$ and let $\Gamma=\rho\left(\pi_{1}(M)\right)$. $\Gamma$ is a discrete 
subgroup of $\operatorname{Isom}\left(\mathbf{H}^{3}\right) \subset \operatorname{Isom}\left(\mathbf{H}^{4}\right)$ isomorphic to $\pi_{1}(M)$ with domain of discontinuity $\Omega$ in $S^{3}$ consisting of the upper and lower hemispheres. $\mathbf{H}^{4} / \Gamma$ is a complete hyperbolic 4-manifold and $\left(\mathbf{H}^{4} \cup \Omega\right) / \Gamma$ is homeomorphic to $M \times I$ where $I$ is the closed unit interval.

If $S_{+}^{3}$ is the upper hemisphere of $S^{3}$, then $S_{+}^{3}$ also acts as a model for $\mathbf{H}^{3}$ with the same Möbius group acting as the group of isometries. Using $S_{+}^{3}$ as the model for $\mathbf{H}^{3}$, the developing map takes $\tilde{M}$ conformally to $S_{+}^{3} \cdot \operatorname{dev}(\tilde{\Delta})$ is then a Möbius triangulation of $S_{+}^{3}$ in the sense of [16], invariant under $\Gamma$. (A Möbius triangulation is a triangulation where all the faces are parts of 2-spheres).

Let $\tilde{T}$ be a Möbius tetrahedron in $\operatorname{dev}(\tilde{\Delta})$. There is a unique two sphere $S^{2}$ in $S^{3}$ passing through the four vertices of $\tilde{T}$, this bounds a complete totally geodesic hyperplane, denoted by $H(\tilde{T})$, in $\mathbf{H}^{4}$. The convex hull of the four vertices of $\tilde{T}$ in $\mathbf{H}^{4}$, denoted by $C(\tilde{T})$, is an ideal tetrahedron in $\mathbf{H}^{4}$ lying on $H(\tilde{T})$. If $\tilde{T}_{1}$ and $\tilde{T}_{2}$ are two tetrahedra in $\operatorname{dev}(\tilde{\Delta})$ sharing a common face, then $C\left(\tilde{T}_{1}\right)$ and $C\left(\tilde{T}_{2}\right)$ also share a common face, they lie on two (not necessarily distinct) intersecting hyperplanes in $\mathbf{H}^{4}$ bent at some angle $\theta$, where $\theta=0$ if and only if the vertices of $\tilde{T}_{1}$ and $\tilde{T}_{2}$ all lie on the same two sphere $S^{2}$, see figure 1.

The union of $C(\tilde{T})$ where $\tilde{T}$ runs over all the tetrahedra of $\operatorname{dev}(\tilde{\Delta})$ is therefore a singular pleated hypersurface in $\mathbf{H}^{4}$, denoted by $S_{\rho, \Delta}$, invariant under the action of $\Gamma$. Modulo the action of $\Gamma$, we have $S_{\rho, \Delta} / \Gamma \subset \mathbf{H}^{4} / \Gamma$ is a singular pleated hyperbolic structure on $M$, dependent only on the original hyperbolic structure and the triangulation $\Delta$, we denote it by $M_{\rho, \Delta}$. The points $v_{1}, \ldots, v_{k} \in M_{\rho, \Delta}$ all develop to the sphere at infinity and hence are ideal singular points of codimension 3. This gives part (b) of lemma 1.

We next see what happens when we perturb the point $\left[\rho ; v_{1}, . ., v_{k}\right] \in \mathcal{C}(M, k)$ slightly. First we recall the holonomy theorem (Hejhal, Thurston, Lok, Goldman, etc., see for example [15], [22]) which states that locally, a $(G, X)$ structure on $M$ is determined by its holonomy or equivalently, the holonomy map from the deformation space of $(G, X)$-structures on a manifold $M$ to the space $\operatorname{Hom}\left(\pi_{1}(M), G\right) / G$ is an open map.

Suppose that $\left[\rho^{\prime} ; v_{1}^{\prime}, . ., v_{k}^{\prime}\right] \in \mathcal{C}(M, k)$ is sufficiently close to $\left[\rho ; v_{1}, \ldots, v_{k}\right]$. 


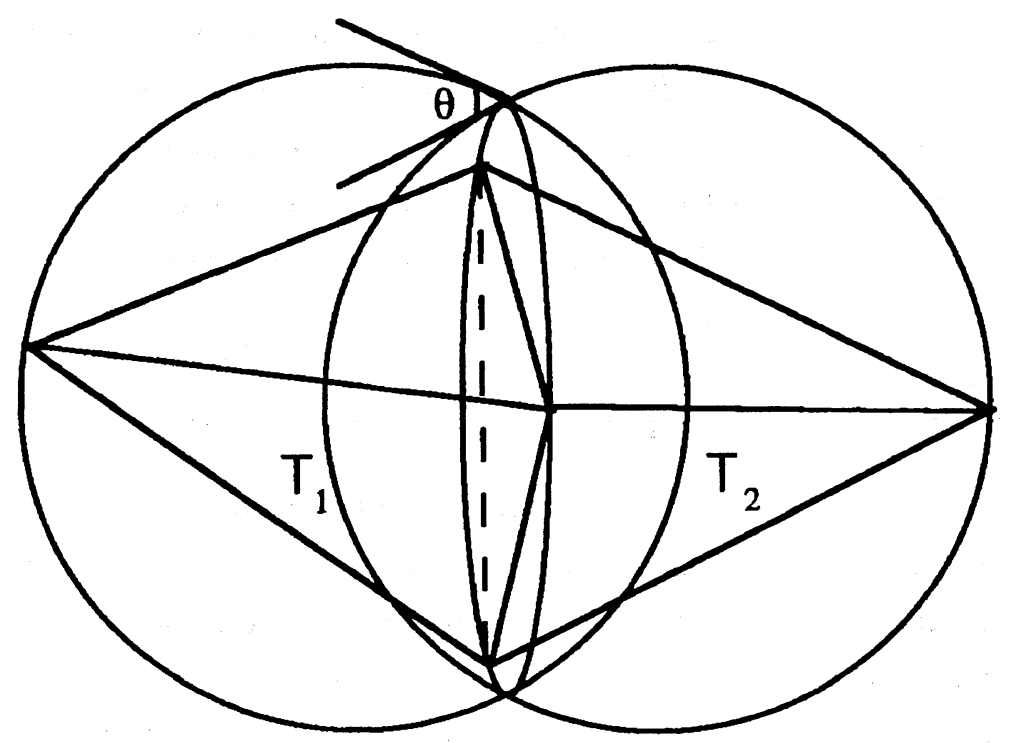

FIGURE 1. Adjacent tetrahedra $T_{1}$ and $T_{2}$ such that $C\left(T_{1}\right)$ and $C\left(T_{2}\right)$ is bent at a positive angle $\theta$ along their common face.

Then $\Gamma^{\prime}=\rho^{\prime}\left(\pi_{1}(M)\right)$ is still a discrete subgroup of $\operatorname{Möb}\left(S^{3}\right)$ isomorphic to $\pi_{1}(M)$ and the limit set of $\Gamma^{\prime}$ is topologically a sphere, by results of D. Sullivan [19], P. Tukia [24], and the holonomy theorem, see [11] for details . $M \cong \Omega_{i} / \Gamma^{\prime}$ where $\Omega_{i}$ is one of the two domains of discontinuity of $\Gamma^{\prime}$ in $S^{3}$. If $\Lambda\left(\Gamma^{\prime}\right)$ is the limit set of $\Gamma^{\prime}$, then $\Omega_{i} \cup \Lambda\left(\Gamma^{\prime}\right)$ is homeomorphic to the closed ball by results of M. Bestvina and G. Mess, see [3]. A triangulation $\Delta^{\prime}$ of $M$ with vertices at $v_{1}^{\prime}, \ldots, v_{k}^{\prime}$ isotopic to $\Delta$ lifts to a triangulation $\widetilde{\Delta^{\prime}}$ of $\tilde{M}$ and hence $\Omega_{i}$ by the developing map. If $\rho^{\prime}$ is not the hyperbolic structure, then such a triangulation can no longer be made Möbius by results of F. Luo [16], nonetheless, for each tetrahedron $\widetilde{T^{\prime}}$ of $\widetilde{\Delta^{\prime}}$, the convex hull of its vertices in $\mathbf{H}^{4}$ will still be an ideal tetrahedron $C\left(\widetilde{T^{\prime}}\right)$ whose complex parameter is close to that of $C(\tilde{T})$. The previous construction goes through and we get a singular pleated hypersurface $S_{\rho^{\prime}, \Delta^{\prime}}$ equivariant under $\Gamma^{\prime}$. Again, modulo the action of $\Gamma^{\prime}$, we have $S_{\rho^{\prime}, \Delta^{\prime},} / \Gamma^{\prime}$ is a singular pleated hyperbolic structure $M_{\rho^{\prime}, \Delta^{\prime}}$ on $M$ close to $M_{\rho, \Delta}$.

Conversely, if we have a singular pleated hyperbolic structure on $M$ satisfying the conditions of (b) in theorem 1 and which is close to $M_{\rho, \Delta}$, then we have a representation $\rho^{\prime}$ of $\pi_{1}(M)$ into $\mathrm{Möb}\left(S^{3}\right)$ close to $\rho$. Again by the holonomy 
theorem and the results of Sullivan and Tukia, this is a quasi-fuchsian representation. Passing to the sphere at infinity, we obtain a quasi-Fuchsian f.c.s. on $M$ together with $k$ distinguished points which are the ideal codimension 3 singular points, thus giving a point $\left(\rho^{\prime} ; v_{1}^{\prime}, \ldots, v_{k}^{\prime}\right) \in \mathcal{C}(M, k)$ close to $\left(\rho ; v_{1}, \ldots, v_{k}\right)$. This gives the local homeomorphism between the spaces described in (a) and (b) of theorem 1.

\section{IDEAL HYPERBOLIC TETRAHEDRA AND EUCLIDEAN POLYHEDRA}

We saw in the previous section that the basic building blocks of the singular pleated hyperbolic structures on $M$ were ideal tetrahedra. To specify the way two tetrahedra are glued together along a face, it suffices to specify the pleating or bending measure along the face. However, to actually obtain a singular pleated hyperbolic structure on $M$, we need to ensure that the holonomy is trivial about the edges. Thus the pleating measures as we go around an edge are not independent, they need to satisfy certain conditions which we shall examine in detail.

We first recall some well-known facts about ideal hyperbolic tetrahedra (see [22] for details). Let $T$ be an ideal tetrahedron in $\mathbf{H}^{3}$ and $L(v)$ the link of an ideal vertex $v$ of $T . L(v)$ is a Euclidean triangle defined up to orientation preserving similarity and $L(v)$ determines $T . L(v)$ can be concretely realised by taking the intersection of $T$ with a horosphere about $v$. It follows that $T$ is determined by the three dihedral angles $\alpha, \beta$ and $\gamma$ of edges incident to the ideal vertex $v$, and that $\alpha+\beta+\gamma=\pi$. Furthermore, the dihedral angles of opposite edges are equal and the oriented similarity class of $L(v)$ does not depend on the choice of the vertex $v$, see figure 2 .

The euclidean triangles up to similarity can be parametrised by complex numbers as follows: To each vertex $v$ of a triangle $\Delta(t, u, v)$ we associate a complex number, namely the ratio $\frac{(t-v)}{(u-v)}=z(v)$ of the sides adjacent to $v$ where the vertices are labelled in clockwise order so that $\operatorname{Im}(z(v))>0$, see figure 3. If $z(v)=z$, it follows that $z(t)=\frac{z-1}{z}$ and $z(u)=\frac{1}{1-z}$. Thus each edge $e$ of the tetrahedron $T$ is labelled with a complex number $z(e)$, opposite edges have the same label, and the label on any one edge determines the rest. The set of ideal tetrahedra with a distinguished pair of oposite edges can be 


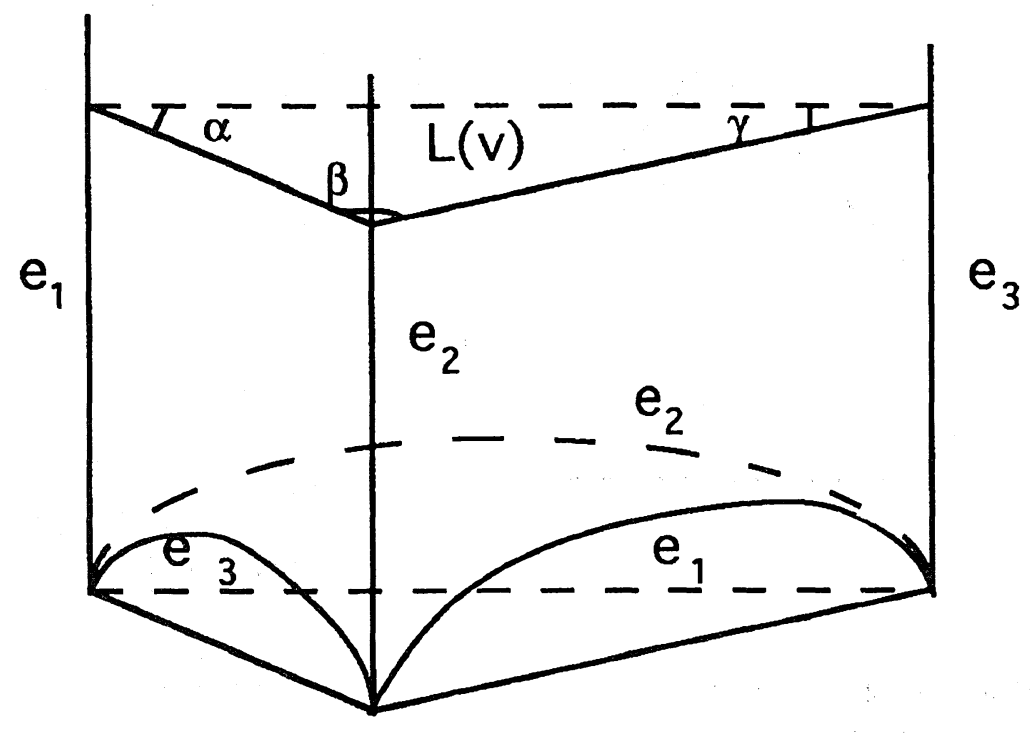

Figure 2. An ideal tetrahedron where $L(v)$ is an $(\alpha, \beta, \gamma)$ triangle.

identified with the complex numbers with positive imaginary part, i.e., the upper half plane.

We now pin down the exact conditions that must be satisfied by the complex parameters $z_{i}$ associated to the tetrahedra $T_{i}$ (with distinguished pair of opposite edges) and the pleating measures $p_{j}$ on the faces $f_{j}$. First, note that if we assign independent complex parameters $z_{i}$ to each of the tetrahedra $T_{i}$ of $\Delta$ and glue the faces together in the combinatorial pattern determined by $M$, we obtain a hyperbolic structure on $M-\{1-$ skeleton $\}$. To ensure that the structure is a cone structure, we must ensure that the holonomy about each edge is either trivial or a pure rotation, i.e., has trivial translation. Let $e$ be an edge of $\Delta$ and let $e_{1}, \ldots, e_{l}$ be the opposite edges of the tetrahedra meeting at $e$ with complex parameters $z\left(e_{1}\right), \ldots, z\left(e_{l}\right)$ respectively (recall that the complex parameters of opposite edges are equal). The algebraic condition that the holonomy about the edges has trivial translation (cf. [22]) is

$$
\left|z\left(e_{1}\right) z\left(e_{2}\right) \ldots z\left(e_{l}\right)\right|=1
$$

If the condition $(*)$ is satisfied about all edges, we obtain a cone hyperbolic structure on $M$ with ideal vertices. To obtain a singular pleated hyperbolic 


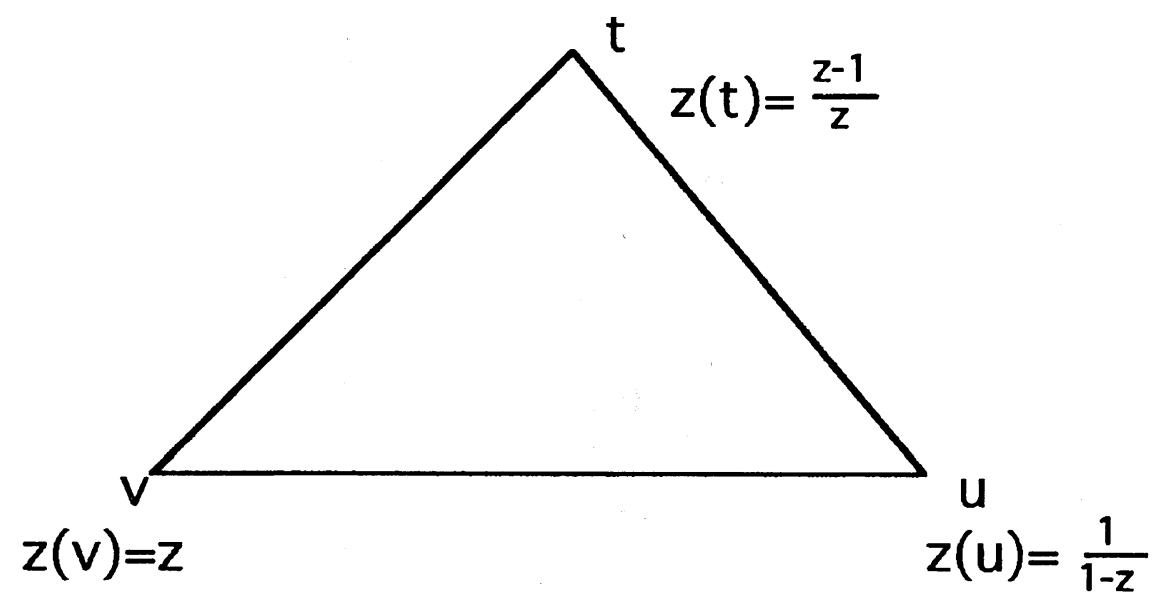

FiguRE 3. Complex parameters associated to the vertices of a euclidean triangle

structure on $M$ with underlying structure the above cone-hyperbolic structure and pleating locus contained in the set of faces of $\Delta$, we also need to specify the pleating measures on the faces. As above, let $e$ be an edge of $\Delta$, $e_{1}, \ldots, e_{l}$ be the edges of the tetrahedra meeting at $e$ with complex parameters $z\left(e_{1}\right), \ldots, z\left(e_{l}\right)$, and $f_{1}, . ., f_{l}$ be the faces meeting at $e$ with pleating measures $p_{1}, . ., p_{l}$ respectively, where $f_{i}$ separates $e_{i}$ and $e_{i+1}$. Also, let $\theta_{i}=\arg \left(z\left(e_{i}\right)\right)$. For parameters which are sufficiently close to those of the singular pleated hyperbolic structure $M_{\rho, \Delta}$, a necessary and sufficient condition that we obtain a singular pleated structure is that the holonomy is actually trivial about each edge (so that we obtain a representation of $\pi_{1}(M)$ into Isom $\left(\mathbf{H}^{4}\right)$ ). Apart from (*) above, this means that the link of each edge $e$, must have the induced structure of a spherical polygon where the sides of the spherical polygon correspond to the edges $e_{i}$ and have length $\theta_{i}$ and the vertices correspond to the faces $f_{i}$ with exterior angles $p_{i}$. The polygon need not be convex but to ensure that the representation actually corresponds to the holonomy of a singular pleated hyperbolic structure, the polygon must be proper, i.e., the sides do not cross. A negative $p_{i}$ corresponds to a negative exterior angle which means that the spherical polygon is not convex about that particular vertex. In general, this gives three independent relations for the parameters $\theta_{1}, \ldots, \theta_{l}, p_{1}, \ldots, p_{l}(\operatorname{cf}[20])$ 
for each edge $e$ of the triangulation $\Delta$.

We finish up the proof of lemma 1 and theorem 1 by showing the correspondence between the respective parts (b) and (c). The singular pleated hyperbolic structures on $M$ satisfying the conditions of part (b) induce singular pleated euclidean structures up to similarity on the links of the vertices $L\left(v_{i}\right)$. To see this, take the developing image and look at the intersection of $L\left(v_{i}\right)$ with a horosphere in $\mathbf{H}^{4}$. Each link is a euclidean polyhedron up to similarity and clearly must satisfy the conditions of part (c) of theorem 1. Conversely, given a set of euclidean polyhedra $P_{1}^{\prime}, \ldots, P_{k}^{\prime}$ close to $P_{1}, \ldots, P_{k}$ satisfying the conditions of (c) of theorem 1 , we can construct (uniquely) a singular pleated hyperbolic structure on $M$ satisfying the conditions of (b) with ideal tetrahedra building blocks and bending measures specified by the dihedral angles of the euclidean polyhedra since the condition $(*)$ and the spherical polygonal condition about the edges are equivalent to the fact that the faces of the euclidean polyhedra $P_{1}^{\prime}, \ldots, P_{k}^{\prime}$ close up nicely about the vertices. This completes the proof of theorem 1 .

We conclude by doing a crude dimension count. Each tetrahedron $T_{i}$ of $\Delta$ gives one complex parameter, this gives $2 m$ real parameters since there are $m$ tetrahedra. There are also $2 m$ faces with $2 m$ real pleating parameters so that there are altogether $4 m$ real parameters. There are $m+k$ edges and for each edge, there is one relation arising from the cone-hyperbolic structure condition $(*)$ and three relations from the spherical polygonal conditions that ensures that the holonomy about the edge is trivial. This gives $4(m+k)$ relations. Note however that if $v$ is a vertex and $e_{1}, \ldots, e_{l}$ are edges ending at $v$, and if the holonomy around $e_{1}, \ldots, e_{l-1}$ is trivial, then the holonomy around $e_{l}$ is also trivial so that we can subtract a set of 4 relations for each vertex. This still leaves $4 m$ relations. However, since $\mathcal{C}(M, k)$ has dimension at least $3 k$, the $4 m$ relations cannot be independent (compare with [22] where similar parameters and relations were obtained when finding the deformation space of hyperbolic structures on a knot complement). It seems that we need to study the equations arising from the specific cases more carefully if we are to obtain qualitative information on the local structure of $\mathcal{C}(M)$.

Finally, we note that in studying singular pleated structures on $M$ relative 
to $\Delta$, we could have used as building blocks finite hyperbolic tetrahedra. In this case, the cone hyperbolic structure is parametrised by the length of the edges, there are still pleating parameters along the faces of $\Delta$ but now the corresponding space would be $\mathcal{C}(M, k) \times \mathbb{R}^{k}$. Much of the arguments of [21] (where the two-dimensional case was dealt with) would carry through, we refer to the details to the reader.

\section{REFERENCES}

1. Apanasov, B., Nontriviality of Teichmüller Space for Kleinian groups in space, Riemann surfaces and related Topics: Proceedings of the 1978 Stony Brook Conference, I. Kra and B. Maskit (editors), Ann. of Math. studies 97, Princeton University Press 1981, 21-31.

2. Apanasov, B., Deformations of Conformal Structures on hyperbolic manifolds, J. Differential Geometry 35 (1992), 1-20.

3. Bestvina, M. and Mess, G., The boundary of negatively curved groups, J. of Amer. Math. Soc. 4:3 (1991).

4. Epstein, D.P.A. and Marden, A., Convex hulls in hyperbolic spaces, a theorem of Sullivan, and measured pleated surfaces, London Mathematical Society Lecture Notes 111 (1987), 114-253.

5. Goldman, W.M., Projective structures with Fuchsian holonomy, J. of Diff. Geom. 25 (1987), 297-326.

6 .

7. Johnson, D. and Millson, J.J., Deformation spaces associated to compact hyperbolic manifolds, in Discrete Groups in Geometry and analysis, Papers in Honor of G.D. Mostow on His Sixtieth Birthday R. Howe (ed.), Progress in Math. 67, Birkhauser, Boston-Basel-Stutgart 1987, 48-106.

8. Kamishima, Y., Conformally flat manifolds whose development maps are not surjective, Trans. Amer. Math. Soc. 294 (1986), 607-623.

9. Kamishima, Y. and Tan, S.P., Deformation Spaces associated to Geometric Structures, Advanced Studies in Pure Mathematics 20 (1992), 263-300.

10. Kapovich, M.E., Deformations of representations of discrete subgroups of $S O(3,1)$, I.H.E.S. preprint 1990.

11. Kapovich, M.E., Topological Aspects of Kleinian Groups in Several Dimensions, M.S.R.I. preprint 1992.

12. Kourouniotis, C., Deformations of hyperbolic structures on manifolds of several dimensions, Math. Proc. Camb. Phil. Soc. 98 (1985), 247-261.

13. Kulkarni, R.S. and Pinkall, U., Uniformisation of geometric structures with applications to conformal geometry, in Differential Geometry, Peniscola 1985, A.M. Naviera (ed.) Lecture Notes in Math. Springer-Verlag 1209 (1986), 190-210.

14. Kulkarni, R.S. and Pinkall, U., A Canonical Metric for Mobius Structures and Its Applications, preprint.

15. Lok, L., Deformations of locally homogeneous spaces, Ph.D. thesis, Columbia University 1984. 
16. Luo, F., Triangulations in Möbius geometry, to appear in Transactions of the Amer. Math. Soc.

17. Matsumoto, S., Foundations of flat conformal structures, Advanced studies in pure Mathematics 20 (1992), 167-261.

18. Mostow, G.D., Quasiconformal mappings in $n$-space and the rigidity of hyperbolic space forms, Publ. Math. Inst. Hautes Etudes Sci. 34 (1968), 53-108.

19. Sullivan, D., Quasiconformal homeomorphisms and dynamics: Structural stability implies hyperbolicity, Acta Math. 155 (1985), 243-260.

20. Tan, S.P., Deformations of flat conformal structures on a hyperbolic 3-manifold, J. of Diff. Geom. 37 (1993), 161-177.

21. Tan, S.P., Singular pleated surfaces and $\mathbf{C P}^{1}$-structures, to appear in Glasgow Math. Journal.

22. Thurston, W.P., The Geometry and Topology of three-manifolds, Princeton University Mathematics Department notes 1979.

23. Thurston, W.P., Shapes of Polyhedra, Research Report GCG 7, Geometry Supercomputer Project.

24. Tukia, P., On isomorphisms of geometrically finite groups, Publ. of I.H.E.S. 61 (1985).

National University of Singapore, Singapore

E-mail address: mattansp@nusvm.bitnet or mattansp@nusunix.nus.sg

RECEIVED FEBRUARY 10, 1994. 